\title{
Expression analyses of three members of the AtPHOI family reveal differential interactions between signaling pathways involved in phosphate deficiency and the responses to auxin, cytokinin, and abscisic acid
}

\author{
Cécile Ribot • Yong Wang • Yves Poirier
}

Received: 23 November 2007 / Accepted: 30 November 2007 / Published online: 19 December 2007

(C) Springer-Verlag 2007

\begin{abstract}
The PHO1 protein is involved in loading inorganic phosphate $(\mathrm{Pi})$ to the root xylem. Ten genes homologous to AtPHOI are present in the Arabidopsis thaliana (L.) Heyn genome. From this gene family, transcript levels of only AtPHOl, AtPHOl;H1 and AtPHOl;H1O were increased by Pi-deficiency. While the up-regulation of AtPHOl;H1 and AtPHOl;H1O by Pi deficiency followed the same rapid kinetics and was dependent on the PHR1 transcription factor, phosphite only strongly suppressed the expression of AtPHOl;Hl and had a minor effect on AtPHOI;H1O. Addition of sucrose was found to increase transcript levels of both AtPHOI and AtPHOl;HI in Pi-sufficient or Pi-deficient plants, but to suppress AtPHO1:H1O under the same conditions. Treatments of plants with auxin or cytokinin had contrasting effect depending on the gene and on the Pi status of the plants. Thus, while both hormones down-regulated expression of AtPHOI independently of the plant Pi status, auxin and cytokinin up-regulated AtPHOl;HI and AtPHOl;H1O expression in Pi-sufficient plants and down-regulated expression in Pi-deficient plants. Treatments with abscisic acid inhibited AtPHOI and AtPHOl;HI expression in both Pi-sufficient and Pi-deficient plants, but increased AtPHO1;H1O expression under the same conditions. The inhibition of expression by abscisic acid of AtPHOl and AtPHOl; $H 1$, and of the Pi-starvation responsive genes AtPHT1;1 and AtIPS1, was dependant on the ABI1 type 2C protein phosphatase. These results reveal that various levels of cross talk between the signal transduction pathways to
\end{abstract}

C. Ribot $\cdot$ Y. Wang $\cdot$ Y. Poirier $(\bowtie)$

Department of Plant Molecular Biology,

Biophore Building, University of Lausanne,

1015 Lausanne, Switzerland

e-mail: yves.poirier@unil.ch
$\mathrm{Pi}$, sucrose and phytohormones are involved in the regulation of expression of the three AtPHOI homologues.

Keywords Abscisic acid - Arabidopsis · Auxin ·

Cytokinin $\cdot$ PHO1 $\cdot$ Phosphate $\cdot$ PHR1

\begin{tabular}{|c|c|}
\hline \multicolumn{2}{|c|}{ Abbreviations } \\
\hline 2,4-D & 2,4-Dichlorophenoxy-acetic acid \\
\hline $\mathrm{ABA}$ & Abscisic acid \\
\hline ABI1 & Abscisic acid insensitive 1 \\
\hline $\mathrm{Pi}$ & Inorganic phosphate \\
\hline PHR1 & Phosphate starvation response 1 \\
\hline PHO1 & Phosphate deficient 1 \\
\hline
\end{tabular}

\section{Introduction}

Phosphorus is one of six essential macronutrients that plants acquire from the soil and utilize for their growth and development. Although the total amount of phosphorus in the soil may be high, inorganic phosphate $(\mathrm{Pi})$, the main form of phosphorus assimilated by plants, is relatively inaccessible to plant roots because of its low solubility and high-sorption capacity in soil. This inaccessibility constitutes one of the major constraints for plant growth in natural ecosystems. To maintain phosphorus homeostasis within critical limits for optimal development, plants have evolved various systems that regulate $\mathrm{Pi}$ acquisition from the soil solution and its distribution to different organs and cellular compartments (Raghothama 2000; Poirier and Bucher 2002).

Until now, five protein families, implicated in phosphate transport have been identified in plants (Poirier and Bucher 2002). The PHT1 family encodes high-affinity proton-coupled Pi transporters primarily expressed in plant roots. The 
characterization of loss-of-function A. thaliana mutants in the AtPHT1;1 and AtPHT1;4 genes showed a strong reduction in Pi-uptake capacity, demonstrating the major role of these transporters in phosphate absorption into roots (Misson et al. 2004; Shin et al. 2004). After penetrating in root cells, acquired Pi needs to be distributed to the various tissues of the plant as well as to the different organelles of the cells. Whereas no phosphate transporter has yet been identified in the tonoplast, specific transporters have been localized to membranes of plastids and mitochondria. These include the plastid PHT2;1 thought to function as a $\mathrm{H}^{+} / \mathrm{Pi}$ symporter, several types of plastidic phosphate translocators acting as phosphate/sugar exchangers, and PHT3 acting as a mitochondrial $\mathrm{Pi} / \mathrm{H}^{+}$symporter (Poirier and Bucher 2002).

Proper distribution of $\mathrm{Pi}$ among the various plant tissues requires the loading and unloading of $\mathrm{Pi}$ in the xylem and phloem. The A. thaliana AtPHOl gene has been demonstrated to have a crucial role in Pi loading to the root xylem vessel. Physiological characterization of the phol mutant showed that only $3-10 \%$ of the wild-type level of Pi was translocated to shoots while Pi uptake into the root was unaffected in the mutant (Poirier et al. 1991). AtPHO1 has a large hydrophilic $\mathrm{N}$-terminus and a $\mathrm{C}$-terminus containing a minimum of six putative transmembrane domains (Hamburger et al. 2002). AtPHO1 is predominantly expressed in the stellar cells of the root and of the lower part of the hypocotyl and the protein shares no sequence homology with any characterized solute transporters (Hamburger et al. 2002). Although the precise mode of action of AtPHO1 remains to be elucidated, it is clear that the protein plays an important role in phosphate homeostasis. The A. thaliana genome contains ten additional genes encoding proteins having significant sequence similarity and the same topology to AtPHO1, which consequently form a novel class of proteins likely involved in plant Pi transport (Wang et al. 2004). Although the promoters of a majority of AtPHOl family members are active in the vascular tissue, such as AtPHOl and AtPHOl;H1, some members have distinct expression pattern, such as AtPHO1;H9, expressed in the pollen and AtPHO1;H1O expressed in the root epidermal/ cortical cells (Wang et al. 2004). It has recently been shown that among the homologues of AtPHOl, only one gene, namely AtPHOl;H1, can complement the phol mutant when expressed under the control of the $\mathrm{PHOl}$ promoter, thus revealing a limited functional redundancy for Pi loading to the root xylem among the members of the AtPHOl gene family (Stefanovic et al. 2007).

Over the past 5 years, several studies aimed at analyzing the pattern of gene expression using microarrays have revealed a complex network of genes that are up- and down-regulated at various points following $\mathrm{Pi}$ starvation either in roots or in shoots (Wang et al. 2002; Uhde-Stone et al. 2003; Wu et al. 2003; Misson et al. 2005; Morcuende et al. 2007; Müller et al. 2007). At present, relatively little is known of the components of the signal transduction cascade(s) involved in sensing Pi nutrient status and triggering a coordinated adaptive response to Pi deficiency in plants. The transcription factors PHR1, WRKY75 and BHLH32 in A. thaliana, and OsPTF1 in rice, as well as the small ubiquitin-related modifier E3 ligase SIZ1 in A. thaliana, have been identified as participating in the response of plants to Pi deficiency (Rubio et al. 2001; Miura et al. 2005; Yi et al. 2005; Chen et al. 2007; Devaiah et al. 2007). The At4 and IPS1 genes and the micro RNA miR399, which are all strongly induced upon Pi starvation, have been implicated in controlling the expression of $\mathrm{PHO} 2$, a gene encoding a ubiquitin-conjugating E2 enzyme playing an important role in phosphate distribution homeostasis under Pi deficiency (Aung et al. 2006; Bari et al. 2006; Chiou et al. 2006; Shin et al. 2006; Franco-Zorilla et al. 2007). LTR1, a gene encoding a multicopper oxidase, has been implicated in the reduction of primary root growth upon Pi-deficient conditions (Svistoonoff et al. 2007).

Other factors have been found to influence the responses of plants to Pi deficiency. Recent studies have indicated potential interactions between Pi-deficiency signaling and sugar-sensing pathways. Sucrose has been implicated in the transcriptional control of several genes induced upon Pi deficiency, such as the UDP glucose phosphorylase, AtIPS1 (Martin et al. 2000), AtACP5 (encoding an acid phosphatase) and members of the AtPHT1 family, and phosphate deficiency typically leads to high starch and sucrose levels in shoots (Ciereszko et al. 2005; Franco-Zorrilla et al. 2005; Müller et al. 2005, 2007; Karthikeyan et al. 2006). Both hexokinase-dependent and hexokinase-independent signaling pathways are thought to be involved in the interactions between sugar sensing and Pi starvation responses (Müller et al. 2005; Karthikeyan et al. 2006). Several phytohormones have also been implicated in the response and adaptation of plants to $\mathrm{Pi}$ deficiency. For example, both auxin and ethylene have been implicated in modulating the developmental adaptations of roots to Pi deficiency (LópezBucio et al. 2002; Lopez-Bucio et al. 2005; Nacry et al. 2005). Microarray studies from Pi deficient plants have also revealed changes in transcripts levels of genes involved in hormone synthesis or responding to them (Wang et al. 2002; Hammond et al. 2003; Uhde-Stone et al. 2003; Wu et al. 2003; Misson et al. 2005; Morcuende et al. 2007; Müller et al. 2007). Cytokinin and its receptor CRE1 have been found to play an important role in suppressing the upregulation of several genes following Pi deficiency (Martin et al. 2000; Franco-Zorrilla et al. 2002, 2005; Karthikeyan et al. 2002; Hou et al. 2005; Wang et al. 2006), implicating a cross talk between $\mathrm{Pi}$ and cytokinin signal transduction pathways. 
The goal of this work was first to analyze the pattern of expression of the members of the AtPHOI gene family under Pi-deficiency. The influence of sugar and of the phytohormones auxin, cytokinin and abscisic acid on the expression $\mathrm{AtPHOl}$ gene family members responding to Pi-deficiency was then studied to reveal common patterns of regulation among these genes, as well as potential level of cross talk between various signal transduction pathways and the Pi-deficiency response.

\section{Materials and methods}

\section{Plant culture and treatments}

Seeds of wild-type A. thaliana Columbia ecotype, wildtype A. thaliana Landsberg erecta ecotype, phol-3, phrl, pho2, abal-3 and abil-1 mutants were grown aseptically, under continuous light $\left(100 \mu \mathrm{mol} \mathrm{m} \mathrm{m}^{-2} \mathrm{~s}^{-1}\right)$ at $20^{\circ} \mathrm{C}$, on agar-solidified media containing $1 \mathrm{mM} \mathrm{KK_{2 }} \mathrm{PO}_{4}$ (pH 5.5), $5 \mathrm{mM} \mathrm{KNO}{ }_{3}, 20 \mathrm{mM} \mathrm{NH} \mathrm{NO}_{3}, 2 \mathrm{mM} \mathrm{MgSO}_{4}, 1 \mathrm{mM}$ $\mathrm{CaCl}_{2}, 0.1 \mathrm{mM}$ Fe-EDTA, $50 \mu \mathrm{M} \mathrm{H}_{3} \mathrm{BO}_{4}, 12 \mu \mathrm{M} \mathrm{MnSO}_{4}$, $1 \mu \mathrm{M} \mathrm{ZnCl}, 1 \mu \mathrm{M} \mathrm{CuSO}{ }_{4}, 0.2 \mu \mathrm{M} \mathrm{Na}_{2} \mathrm{MoO}_{4}$ and $1 \%$ sucrose. Seeds from pho2, abal-3 and abil-1 mutants were obtained from the Nottingham Arabidopsis Stock Centre (University of Nottingham, UK). For phosphate-deficient media, $\mathrm{KH}_{2} \mathrm{PO}_{4}$ was replaced by $\mathrm{KNO}_{3}$. For phosphite treatment, a phosphite solution of $1 \mathrm{mM}$ prepared from phosphorous acid (Aldrich Chemicals) and $\mathrm{KOH}$ (final $\mathrm{pH}$ of 5.5) was added to media. Stock solutions of kinetin and $( \pm)$-cis, trans-abscisic acid (ABA) were prepared in water (5 and $100 \mathrm{mM}$, respectively) with a drop of $\mathrm{NaOH} 1 \mathrm{~N}$ to help the dissolution, while 2,4-dichlorophenoxy-acetic acid (2,4-D) was dissolved in $100 \%$ ethanol to a concentration of $10 \mathrm{mM}$. All phytohormones were purchased from Sigma (St. Louis, MO, USA).

\section{RNA isolation and Northern-hybridization analysis}

Total RNA was extracted from plant tissues by phenol:chloroform separation and lithium chloride precipitation followed by washes with sodium acetate and ethanol as previously described (Stefanovic et al. 2007). Northern analysis was performed by separating $25 \mu$ gof total RNA on agarose gels containing formaldehyde, transferring to Nylon membranes (Hybond N+; Amersham Biosciences, Little Chalfont, Buckinghamshire, UK) and hybridizing with $\mathrm{P}^{32}$-radiolabelled specific probes according to standard procedures under high-stringency conditions. Specific probes corresponded to GST (Gene Specific Tags) designed in the microarray CATMA project (Crowe et al. 2003) amplified by PCR with specific set of primers or full-length cDNA.

\section{RT-PCR analysis}

Analysis of the expression profile of the AtPHOI family genes was done by semi-quantitative RT-PCR as described in Wang et al. (2004). Pilot experiments were performed on reverse-transcribed products of several genes to identify the number of cycles corresponding to the linear phase of amplification. Based on these results, a protocol based on 20 cycles of PCR amplifications was done for all genes. The oligonucleotides used for primers were designed to amplify a fragment of $400-600$ bases at the $3^{\prime}$ end of the gene. The region amplified encompassed at least two exons, so that PCR fragments generated from contaminating genomic DNA could easily be distinguished from fragments amplified from cDNA based on the size of the products. Following RT and PCR amplification, as described above, the products were analyzed by Southern blot using probes derived from each member of the AtPHOI family using standard procedures.

\section{Results}

Distinct dynamic of changes in AtPHOl, AtPHOl;HI and AtPHOI;H1O mRNA levels in response to the availability of $\mathrm{Pi}$ in the medium

Analysis of the expression of the AtPHOI and AtPHOl;HI genes has previously revealed their up-regulation under Pideficient conditions (Wang et al. 2004; Stefanovic et al. 2007). In order to examine whether other members of the AtPHOl gene family were up-regulated under Pi-deficient conditions, semi-quantitative RT-PCR analyses were performed to detect the gene expression pattern of all members of the $\mathrm{PHOl}$ gene family in 7-day-old seedlings grown in medium containing $1 \mathrm{mM} \mathrm{Pi}$ and then transferred either to medium containing $1 \mathrm{mM}$ Pi or no phosphate for an additional 5 days (Fig. 1). Analysis revealed that in addition to AtPHOl and AtPHOl;H1, the steady state mRNA level of AtPHO1;H1O was also elevated by phosphate deficiency. In contrast, the expression level of AtPHOl; $\mathrm{H} 2, \mathrm{H3}, \mathrm{H} 4, \mathrm{H} 5$, $H 7$, and $H 8$ genes was not influenced by 5 days of phosphate starvation, while $\mathrm{AtPHOI} ; \mathrm{H6}$ and $\mathrm{H} 9$ transcripts were undetectable in seedlings at this stage of development, in agreement with previous results indicating that expression of these genes was restricted to flowers (Wang et al. 2004). Consequently, AtPHOI, AtPHOl;HI and AtPHOl;HIO genes are the only three members of the AtPHOl gene family to be up-regulated by phosphate deficiency.

To better understand the dynamic of AtPHOI, AtPHOl;HI and AtPHOl;H1O responses to phosphate starvation, an analysis of the effect of different Pi concentrations as well as a time course of response to Pi deficiency 
Fig. 1 Expression analysis of the members of the AtPHOI gene family during Pi starvation. Semi-quantitative RT-PCR were performed using RNA extracted from whole plants grown first for 7 days in media containing $1 \mathrm{mM} \mathrm{Pi}$ and then transferred to media containing $1 \mathrm{mM} \mathrm{Pi}(+P)$ or without $\mathrm{Pi}(-P)$ for 5 days. DNA fragment obtained by PCR performed on genomic DNA using the same oligonucleotides used for RT-PCR is shown in the last lane (gDNA). This control ensures that the band obtained by RT-PCR cannot be derived from the amplification of genomic DNA that could be present in the RNA preparations

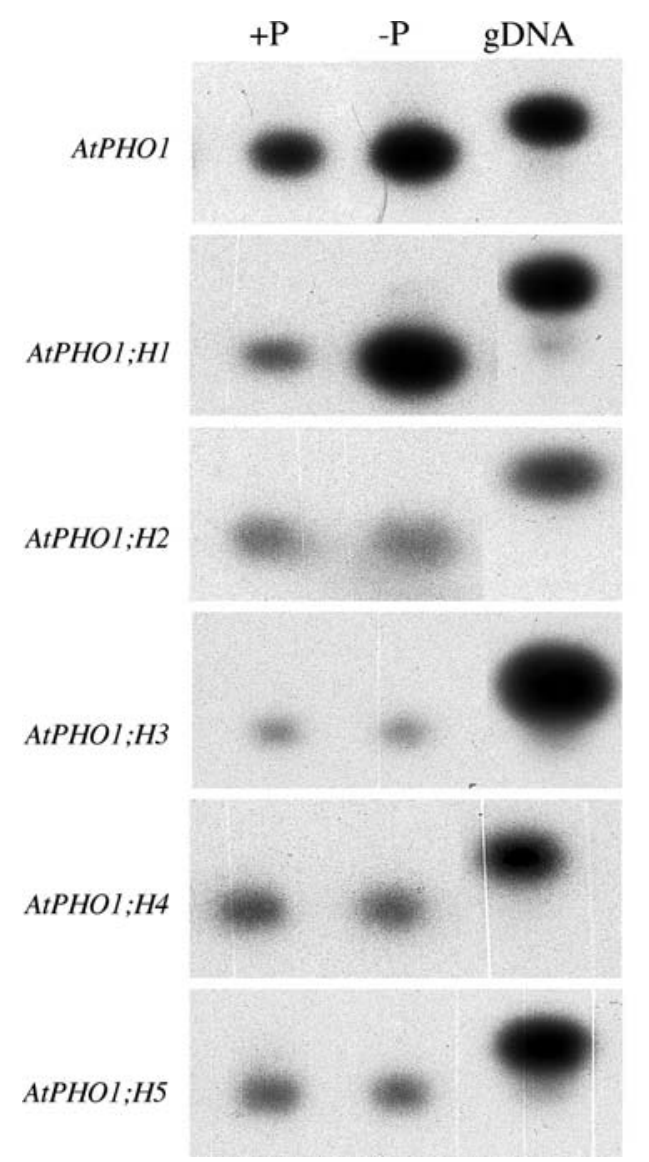

AtPHOI;H6

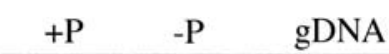

AtPHOI; $H 7$

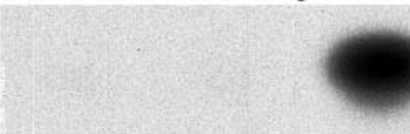

AtPHOI; $H 8$
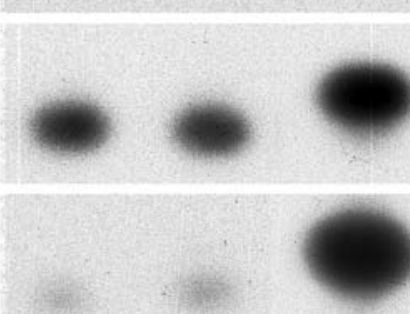

AtPHOI;HIO

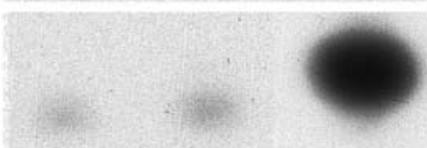

AtPHOI; $\mathrm{H}^{9}$
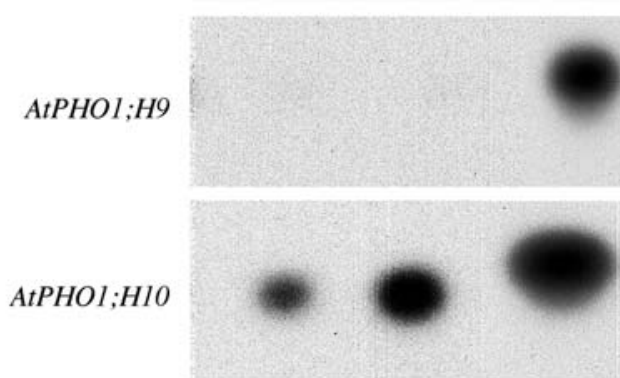

Tubulin $\alpha$

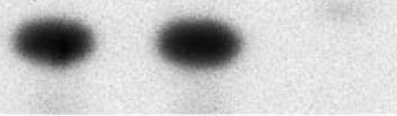

was performed (Fig. 2). Seedlings were first grown for 7 days in medium containing $1 \mathrm{mM} \mathrm{Pi}$ and then transferred for 5 days to media supplemented with $\mathrm{KH}_{2} \mathrm{PO}_{4}$ at concentrations ranging from 0 to $1.25 \mathrm{mM}$. Northern blot analysis showed that the AtPHO1;H1, and AtPHT1;1 transcript levels were slightly more responsive to small decrease in external $\mathrm{Pi}$ concentration compared to AtPHOl and AtPHO1;H10 (Fig. 2a). In contrast, following the transfer of plants to medium without $\mathrm{Pi}$, the increase in mRNA level was clearly more rapid for AtPHOl;H1 and AtPHOl;H1O compared to AtPHOl and AtPHT1;1 (Fig. 2b). Similarly, the down-regulation of mRNA levels following the re-supply of Pi to plants grown in Pi-deficient media was quicker for $A t P H O 1 ; H 1$ and $A t P H O 1 ; H 10$, with a return to basal expression level after $8 \mathrm{~h}$ of $\mathrm{Pi}$ re-supply, compared to AtPHOl and AtPHT1;1 which returned to basal expression level only between 24 and 48 h after Pi re-supply (Fig. 2c).

Effects of phosphite and of the phrl, phol and pho2 mutations on AtPHO1, AtPHO1;H1 and $\mathrm{AtPHO} ; \mathrm{HIO}$ expression

The orthophosphite anion $\left(\mathrm{H}_{2} \mathrm{PO}_{3}{ }^{-}\right.$or $\left.\mathrm{HPO}_{3}{ }^{2-}\right)$ is a nonmetabolizable analogue of phosphate that is able to suppress several typical molecular and developmental responses to Pi limitation (Ticconi et al. 2001; Varadarajan et al. 2002; Kobayashi et al. 2006). Phosphite had previously been shown to strongly suppress the up-regulation of AtPHO1;H1 induced by $\mathrm{Pi}$ deficiency but to have little influence on the expression of AtPHOl under the same conditions (Stefanovic et al. 2007). The induction of AtPHO1;H10 following Pi deficiency was only weakly suppressed by phosphite (Fig. 3a).

The PHR1 transcription factor was found to bind the imperfect palindromic sequence GNATATNC present in promoter regions of several genes whose expression increases during phosphate starvation stress (Rubio et al. 2001). Previous analysis had revealed the presence of the PHR1 binding sequence at position -330 bp from the transcription initiation site in the AtPHOl;Hl gene but not in AtPHO1 (Stefanovic et al. 2007). In agreement with the presence of these sequences, the upregulation of mRNA transcript of AtPHOl; $\mathrm{Hl}$ in plants under Pi-deficient conditions was found to be under the control of PHR1, while this was not the case for the AtPHOl (Fig. 3b; Stefanovic et al. 2007). The AtPHO1; $H 10$ promoter region contains at position -996 bp upstream of the start codon a motif similar to the PHR1 element but not identical in that the first base is a $\mathrm{T}$ instead of a G. However, the sequence TCATATGC retains the structure of an imperfect palindromic sequence. 
Fig. 2 Effect of Pi concentration and kinetic of response of AtPHOl, AtPHOl; Hl, and AtPHO1;H1O gene expression. a Plants grown for 7 days in media containing $1 \mathrm{mM} P \mathrm{Pi}$ were transferred to media containing varying concentrations of Pi $(0,5,10,25,50,125,250$, 500 and $1,250 \mu \mathrm{M}$ ) for 5 days. b Plants grown for 7 days in media containing $1 \mathrm{mM} \mathrm{Pi}(+P)$ were transferred to media without $\mathrm{Pi}(-P)$ for $12,24,48 \mathrm{~h}$, or 3 , 4 , and 5 days. c Plants grown either grown for 10 days in media containing $1 \mathrm{mM} \mathrm{Pi}(+P)$ or grown first for 7 days in media containing $1 \mathrm{mM} \mathrm{Pi}$, transferred for 3 days on Pi-free media $(-P)$, and then transferred to media with $1 \mathrm{mM} \mathrm{Pi}(R)$ for 8,24 or $48 \mathrm{~h}$. For all panels, total RNA was isolated from whole plants and analyzed by Northern-blot analysis

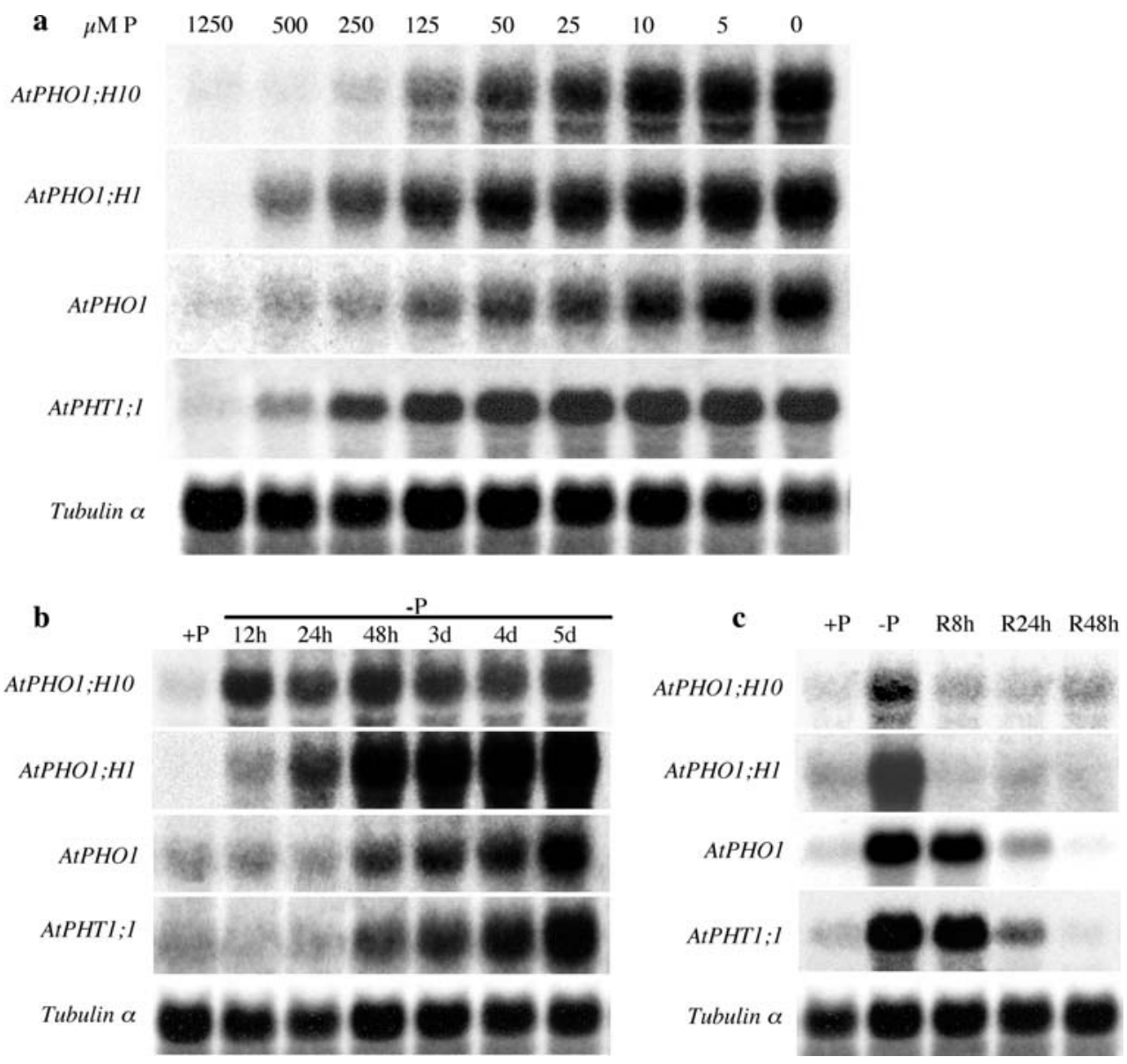

Similar to AtPHOl; Hl, the up-regulation of AtPHO-H1O gene expression observed in Pi-deficient wild-type plants was strongly diminished in the Pi-deficient phrl mutant (Fig. 3b). A similar strong reduction in gene expression under Pi deficiency was also observed for the AtIPSI gene, which also contains a PHR1 binding site, but not for AtPHT1;1, which does not have a PHR1 binding site.

The regulation of AtPHOl, AtPHOl;HI and AtPHOI; $H 10$ mRNA levels was evaluated in phol, a mutant deficient in the transport of Pi to the shoot, and pho2, a mutant over accumulating Pi in the shoot (Poirier et al. 1991; Delhaize and Randall 1995). Both AtPHOl;HI and AtPHOl;H1O transcripts were slightly over accumulated in the shoot of phol mutant compared to wild-type plants grown in solid media containing $1 \mathrm{mM} \mathrm{Pi}$ (Fig. 3c). The expression profile of AtIPS1 in phol also showed a slight over accumulation in leaves, indicating a weak activation of the Pi-starvation signaling pathway in leaves of the mutant grown under high Pi conditions. However, AtPHT1;1 mRNAs level appeared less sensitive to Pi content in phol leaves as the level of expression was unchanged in phol plants grown in Pi-replete media. Under Pi-deficient conditions, all genes tested were up-regulated and no differences were observed between phol and wild-type plants.
For pho2 mutant grown under Pi-sufficient conditions, a slight over-expression of AtPHO1;H1O was observed in leaves while the level of $\mathrm{AtPHOI}$ and $\mathrm{AtPHOI} ; \mathrm{Hl}$ remained unchanged in roots and shoots (Fig. 3d). In contrast, the level of AtIPS1 transcript in pho2 roots was increased. However, under Pi-deficient condition, no difference was found between $p h o 2$ and wild-type for all genes studied.

\section{Regulation of AtPHOl, AtPHOl;HI and AtPHOI;H1O expression by sucrose}

The regulation of AtPHOl, AtPHOl;H1 and AtPHOI;H1O expression by sucrose was investigated in plants grown under Pi-replete or Pi-deficient conditions (Fig. 4). While addition of $30 \mathrm{mM}$ sucrose lead to a reduced expression of $\mathrm{AtPHOI} ; \mathrm{HIO}$ in plants grown in media with or without $\mathrm{Pi}$, sucrose addition led to an increase in mRNAs for AtPHOl, AtPHOl;H1, AtPHT1;1 and AtIPSI independent of the phosphate status.

Regulation of AtPHOI, AtPHOI;HI and AtPHOI;H1O expression by auxin and cytokinin

The regulation of AtPHOl, AtPHOI;HI and AtPHOI;H1O expression by exogenous auxin $(0.5,10 \mu \mathrm{M} 2,4-\mathrm{D})$ and 


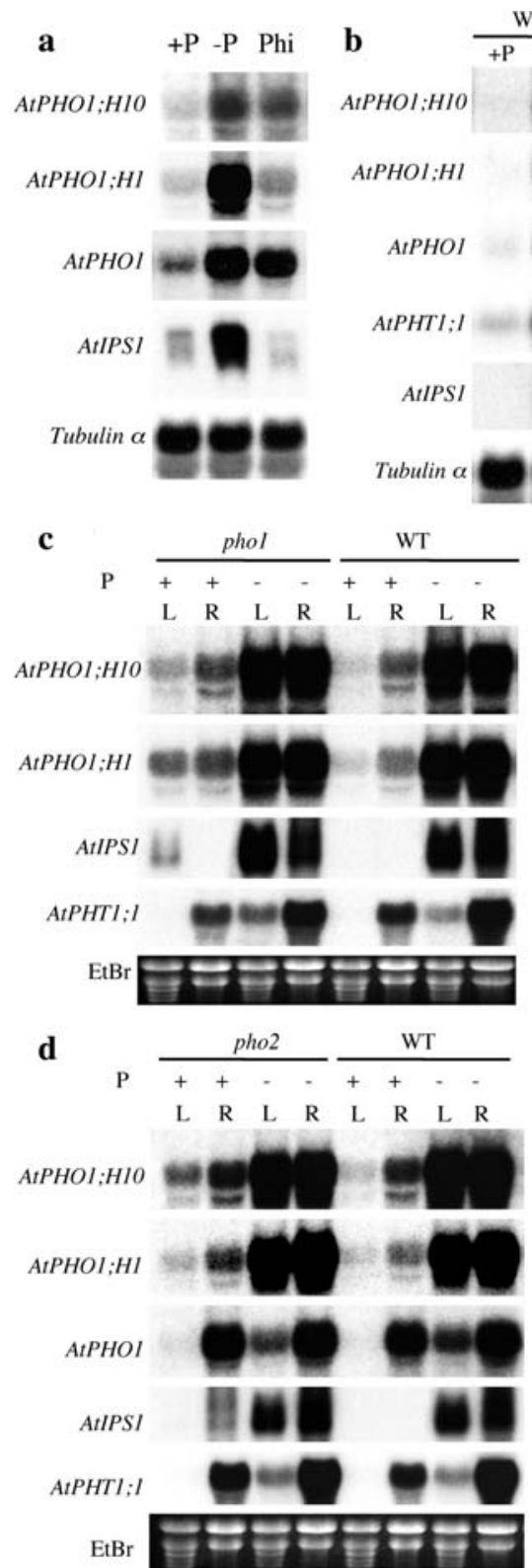

Fig. 3 Effects of phosphite and of the phrl, phol and pho2 mutations on AtPHOl, AtPHO1;H1, and AtPHO1;H1O expression. a Plants grown for 7 days in media containing $1 \mathrm{mM} \mathrm{Pi}$ were transferred to media containing either $1 \mathrm{mM} \mathrm{Pi}(+P)$, no $\mathrm{Pi}(-P)$ or $1 \mathrm{mM}$ phosphite (Phi) for 5 days. Expression profile was compared between wild-type plants and the mutants phrl (b), phol-3 (c), and pho2 (d). For b, c and d, plants grown for 7 days in media containing $1 \mathrm{mM}$ Pi were transferred to media with (+) or without (-) Pi for 5 days. Total RNA (25 $\mu$ gper lane) isolated from either whole plants (b) or separately from leaves $(L)$ of roots $(R ; \mathbf{c}, \mathbf{d})$. For all panels, total RNA was isolated from whole plants and analyzed by Northern-blot analysis

cytokinin (10 $\mu \mathrm{M}$ kinetin) was investigated in plants grown under Pi-replete or Pi-deficient conditions. In plants grown under Pi-deficient conditions, both kinetin and 2,4-D treatments lead to a repression of AtPHOl, AtPHO;HI, AtPHOl;H1O and AtPHT1;1 expression (Fig. 5a, b). In

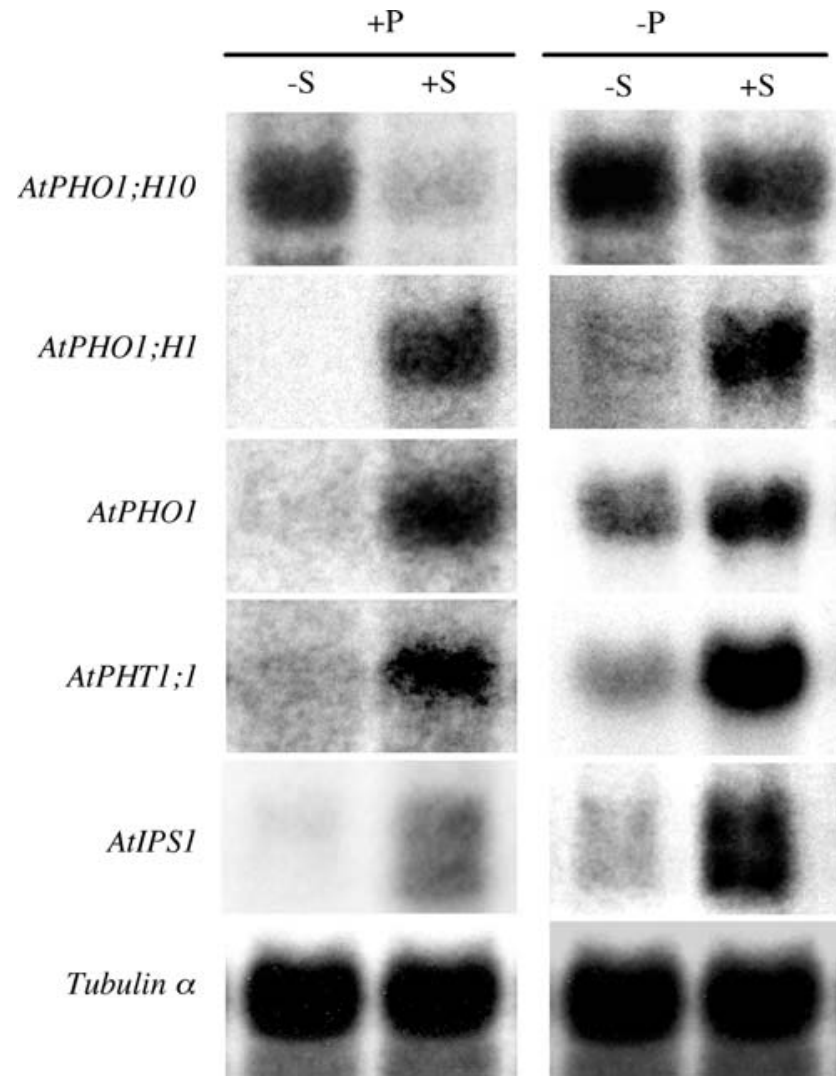

Fig. 4 Effects of sucrose supply on AtPHO1, AtPHO1;H1, and At$\mathrm{PHO} ;$; 10 expression. Plants first grown for 7 days in media without sucrose and with $1 \mathrm{mM}$ Pi before being transferred and grown for an additional 5 days in media containing either $1 \mathrm{mM} \mathrm{Pi}(+P)$ or no $\mathrm{Pi}$ $(-P)$ and supplemented with $30 \mathrm{mM}$ sucrose $(+S)$ or with no added sucrose $(-S)$. Total RNA was isolated from whole plants and analyzed by Northern-blot analysis

contrast, for plants grown under Pi-sufficient conditions, both kinetin and 2,4-D induced the expression of AtPHOl;H1O and AtPHOl;HI but repressed the induction of AtPHOl and AtPHTl;1 (Fig. 5a, b).

Regulation of AtPHOl, AtPHOl;H1 and AtPHOl;H1O expression by abscisic acid

The basal level of expression of the AtPHOl, AtPHOl;H1, AtPHOl;H1O and AtPHTI;1 genes in Pi-sufficient plants was decreased following treatments with abscisic acid (ABA; Fig. 6a). In contrast, the same treatment lead to a sharp increase in AtPHOl; $H 10$ expression (Fig. 6a). The influence of endogenous level of $\mathrm{ABA}$ on the expression of AtPHOl, AtPHOl;HI and AtPHOl;H1O was then examined using the ABA synthesis deficient mutant abal-3 grown under Pi-sufficient condition (Koornneef et al. 1982). While expression of AtPHO1;H1O decreased in the abal-3 mutant compared to wild-type, increase of AtPHOI, AtPHOl;H1 expression was observed in abal-3 (Fig. 6b). 


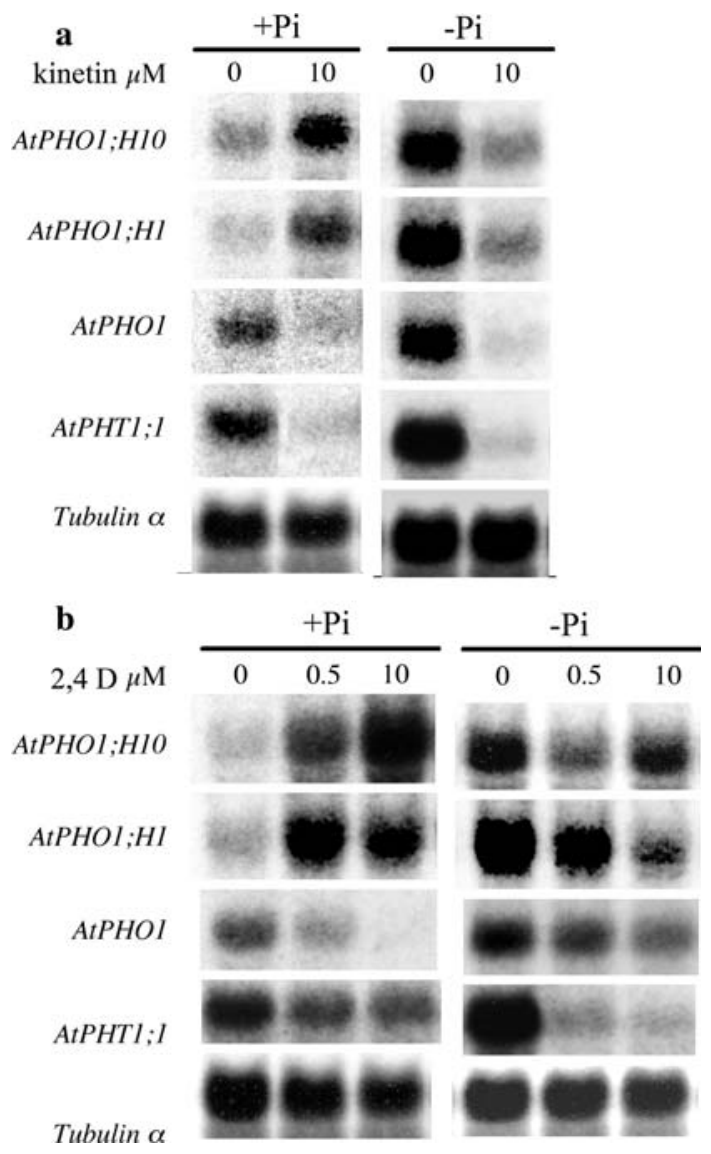

Fig. 5 Effects of cytokinin and auxin treatments on AtPHO1, AtPHO1;H1, and AtPHO1;H1O expression. Plants grown for 7 days in media containing $1 \mathrm{mM}$ Pi were transferred to media containing $1 \mathrm{mM}$ $\mathrm{Pi}(+P)$ or no added $\mathrm{Pi}(-P)$ and supplemented either with the cytokinin kinetin (a) or the auxin 2,4-D (b) for 2 days. Total RNA was isolated from whole plants and analyzed by Northern-blot analysis

The influence of $\mathrm{ABA}$ on the expression of members of the AtPHOl genes in plants under Pi deficiency was examined in wild-type plants and in the ABA-insensitive abil-1 mutant (Koornneef et al. 1982, 1984). In wild-type plants, ABA addition attenuated or abolished the increase of expression of the AtPHOl, AtPHOI;H1, and AtPHTl;1 genes normally observed in Pi-deficient plants, while no such attenuation was observed in the abil-1 mutant (Fig. 6c). Similar results were also obtained for the AtIPSI gene (Fig. 6c). In contrast, addition of ABA to Pi-deficient wild-type plants further enhanced the expression of AtPHO1:10 compared to untreated Pi-deficient plants, and similar results were also obtained for the abil-1 mutant (Fig. 6c).

\section{Discussion}

Of the three $A t P H O 1$ family members that are up-regulated following Pi deficiency, both AtPHOI and AtPHOl;HI have recently been shown to be involved in the transfer of

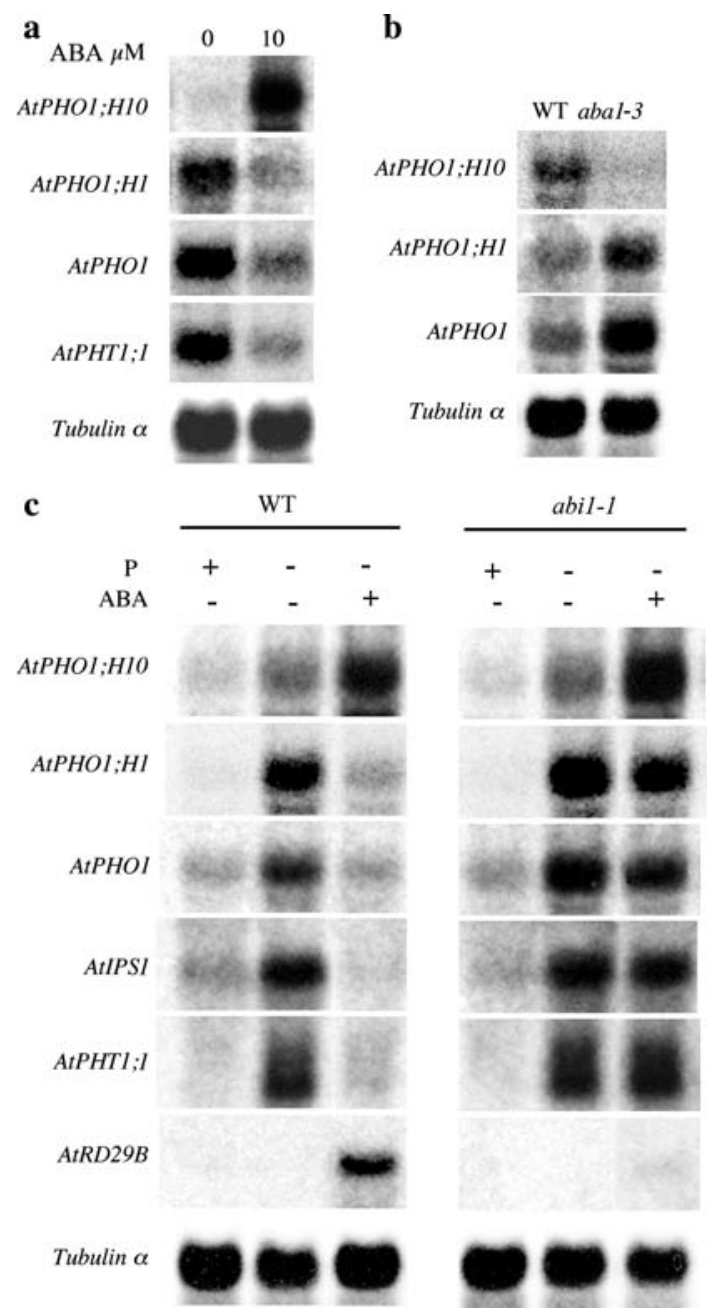

Fig. 6 Effects of ABA on the expression of the AtPHO1, AtPHO1;H1 and $\mathrm{AtPHO1;H1O}$ genes. a Wild-type plants grown for 7 days in media containing $1 \mathrm{mM}$ Pi were transferred to media containing $1 \mathrm{mM} \mathrm{Pi}$ and $10 \mu \mathrm{M}$ ABA for 2 days. b Wild-type plants and abal-3 mutant plants were grown in media with $1 \mathrm{mM}$ Pi for 10 days. $\mathbf{c}$ Wild-type plants and abi 1-1 plants were grown for 7 days in media containing $1 \mathrm{mM} \mathrm{Pi}$, and then transferred to media with (+) or without (-) Pi or ABA $(10 \mu \mathrm{M})$ for 3 days. AtRD29B was used as a control for the expression of a gene induced by $\mathrm{ABA}$ and dependent on ABI1. For all panels, total RNA was isolated from whole plants and analyzed by Northern-blot analysis

Pi to the root vascular cylinder (Stefanovic et al. 2007). Analysis of the single mutants phol and phol;hl as well as the double mutant phol/phol;hl indicated that AtPHO1 has a primary role to play in Pi transfer to the root vascular cylinder, while the contribution of AtPHO1; $\mathrm{H} 1$ to the same transfer was more minor in plants grown either in medium with or without Pi (Stefanovic et al. 2007). The role of AtPHO1;H10 in phosphate homeostasis is unknown but is likely to be distinct from AtPHO1 and AtPHO1; H1. In the phylogenetic tree of AtPHO1 members, AtPHO1;H10 belongs to a clade that is distinct from AtPHO1 and AtPHO1;H1 (Wang et al. 2004). Furthermore, promoter GUS fusions revealed that the AtPHOl;H1O promoter is 
not active in root vascular tissue but rather in root epithelial and cortical cells, making it unlikely that $\mathrm{AtPHO} ; \mathrm{H} 10$ contributes to $\mathrm{Pi}$ transport in or out of the vascular cylinder (Wang et al. 2004).

Previous analysis revealed the presence of two pathways controlling the response of the AtPHOI and AtPHOl;HI genes to Pi deficiency, namely a pathway independent on the PHR1 transcription factor and not influenced by phosphite for AtPHOl, and a distinct pathway dependant on PHR1 and strongly influenced by phosphite for AtPHO1;H1 (Stefanovic et al. 2007). The present work on the regulation of AtPHOl, AtPHO;HI and AtPHOl;H1O provides evidence of further levels of complexity in the pathways involved in the regulation of these members of the AtPHO1 family by Pi-deficiency.

At the level of the kinetic of transcript accumulation following shifts of plants to media with reduced or elevated $\mathrm{Pi}$ concentrations, AtPHOI and AtPHO1;HI behaved differently, with $A t P H O 1 ; H 1$ responding quickly, within 8-12 h, to either a decrease or increase in external Pi level, while AtPHO1 responded more slowly and gradually, typically within $24-48 \mathrm{~h}$ (Fig. 2). The kinetic of transcript changes for AtPHOl; $\mathrm{HlO}$ was very similar to AtPHOl; H1. Morcuende et al. (2007) have recently applied the criteria of a rapid down-regulation of gene expression upon Pi re-supply to Pi-deficient plants in order to distinguish between genes that respond primarily to the Pi status of plants, such as AtPHOl;HI and AtPHOl;H1O, as opposed to genes that may respond more to the secondary effects of Pi-deficiency on metabolism, such as the AtPHOI and AtPHTI;1 genes. Several genes that are strongly up-regulated following $\mathrm{Pi}$ deficiency, such as At4, AtACP5, AtPHT2;1 and AtRNS1 were also found to be quickly down-regulated by Pi re-supply (Müller et al. 2004). The higher responsiveness of the AtPHOl;H1 and AtPHOl;H1O to shift in Pi levels is also consistent with their higher expression in shoots of the phol mutants, which shows reduction of $\mathrm{Pi}$ content in shoots even in plants grown under high external Pi concentrations (Fig. 3c; Poirier et al. 1991; Delhaize and Randall 1995).

An additional feature shared by both AtPHOl;HI and AtPHOI;H1O response to Pi deficiency is their large dependence on the PHR1 transcription factor (Fig. 3b). The phrl mutant of Arabidopsis shows attenuation of several responses associated with Pi-deficiency, including reduced expression of the AtIPS1, At4, AtRNS1 and AtACP5 genes, reduced anthocyanin accumulation and attenuated increase in the root-to-shoot ratio (Rubio et al. 2001). PHR1 was shown to bind to the P1BS (PHR1-binding sequence) motif GNATATNC found in the AtIPS1 promoter. Microarray studies of genes induced under Pi-deficiency have revealed enrichment in genes having the P1BS motif, although numerous genes consistently induced by Pi deficiency did not contain such motif (Wang et al. 2002; Hammond et al. 2003; Uhde-Stone et al. 2003; Wu et al. 2003; Misson et al. 2005; Morcuende et al. 2007; Müller et al. 2007). Although the present study did not identify the sequences within the AtPHO1;H1O regulatory elements to which PHR1 binds, it is likely that PHR1 binds to variants of the P1BS motif, such as potentially the imperfect palindromic sequence TCATATGC found approximately $1 \mathrm{kbp}$ upstream of the start codon, indicating that a larger spectrum of genes may be under the control of PHR1 than previously expected.

One characteristic shared by both AtPHOI and AtPHO1;H1O is the weak effect of phosphite on gene expression following Pi deficiency, in contrast to the strong suppression of AtPHO1;H1 and AtIPS1 expression following phosphite treatment (Fig. 3a). Phosphite is non-metabolized analogue of Pi that is thought to interfere specifically with early events in Pi sensing and signaling and affects a broad spectrum of Pi-starvation responses (Ticconi et al. 2001; Varadarajan et al. 2002). Altogether, these results reveal the existence of partially overlapping regulatory pathways affecting the expression of three members of the AtPHOl gene family in response to Pi deficiency. These include two pathways responding quickly to Pi deprivation, one involving PHR1 and strongly influenced by phosphite regulating AtPHOl;H1, and a second pathway involving PHR1 but weakly influenced by phosphite regulating AtPHO1;H1O. A third pathway responding more slowly to Pi deprivation, that is independent of PHR1 and weakly influenced by phosphite regulates AtPHOI.

Beyond the amount of Pi available to cells, other factors have been shown to influence the response of plants to Pi deficiency, in particular sucrose and phytohormones, such as auxin and cytokinin (Torrey 1976; Franco-Zorrilla et al. 2002; Karthikeyan et al. 2002; Hou et al. 2005; Nacry et al. 2005). The present study reveals that the transcriptional regulation of AtPHOl, AtPHOl;HI and AtPHOl;H1O in response to $\mathrm{Pi}$ status is also influenced by sucrose as well as phytohormones, but in manner that is gene-specific and, in the case of phytohormones, remarkably dependent of the Pi status of the plant.

Transcript level of AtPHOI and AtPHOl;HI as well as of AtPHTI; 1 and AtIPSI were all increased by the addition of sucrose for plants grown either under Pi-replete or Pideficient conditions (Fig. 4). In contrast, under the same conditions, sucrose repressed the accumulation of AtPHOl;H1O transcript (Fig. 4). Promotion of growth of Pi-deficient roots by sucrose addition has been linked to decrease in intracellular $\mathrm{Pi}$ concentration and increased expression of $A t M G D 3$, a gene responsive to Pi deficiency (Lai et al. 2007). Thus, the effect of sucrose on growth and on the Pi demand of cells could also play a role in the regulation of AtPHOI and AtPHOl;H1. However, besides being a metabolite, sucrose is also recognized as a signal 
molecule involved in several physiological responses in plants. An influence of sucrose on Pi-responsive genes as well as an up-regulation of genes involved in carbohydrate metabolism by Pi-deficiency has been demonstrated in several studies (Nielsen et al. 1998; Cieresko et al. 2001; Lejay et al. 2003; Franco-Zorrilla et al. 2005; Müller et al. 2005; Karthikeyan et al. 2006). Recent studies have also demonstrated that several of the morphological responses linked to Pi-deficiency responses are modulated by sucrose, and that numerous genes are synergistically or antagonistically regulated by both sucrose and Pi (Karthikeyan et al. 2006; Müller et al. 2007). The present study thus reveals that AtPHOl and AtPHOl; $\mathrm{Hl}$ genes belong to the group of $\mathrm{Pi}$ starvation responsive genes that are up-regulated by sucrose while AtPHO1;H1O belongs to the group that is down-regulated by sucrose.

Addition of the cytokinin kinetin in the growth medium of phosphate-deficient plants led to the repression of the transcript level of AtPHOI, AtPHOI;HI and AtPHOI;H1O genes (Fig. 5a). These members of the AtPHOl gene family are thus regulated by cytokinin under Pi-deficient conditions in the same way as AtPHT1;1 and many other Piresponsive genes, such as AtIPS1 (Martin et al. 2000; Franco-Zorrilla et al. 2002; Karthikeyan et al. 2002; Hou et al. 2005; Shin et al. 2006). Pi-deficiency has been shown to lead to a decrease in the level of cytokinin that is correlated with altered root morphology (Ei-D et al. 1979; Horgan and Wareing 1980). Addition of cytokinin to Pi-starved roots has also been correlated with an increase in the intracellular Pi content and decrease in growth, thus potentially explaining the repression of Pi-starvation responsive genes by the hormone (Wang et al. 2006; Lai et al. 2007). However, in contrast to Pi-starved plants, addition of cytokinin to Pi-sufficient plants revealed different effects depending on the gene, with transcript accumulation for $\mathrm{AtPHO} ; \mathrm{H} 10$ and $\mathrm{AtPHOl} ; \mathrm{HI}$, and transcript decrease for $\mathrm{AtPHOl}$ and AtPHT1;1 (Fig. 5a). These data reveal an impact of cytokinin on gene expression that likely goes beyond its effect on cell growth and intracellular Pi content and that is influence by the Pi status of the plant cell.

The effects of addition of the auxin 2,4-D were similar to cytokinin, in that auxin treatment suppressed the induction of AtPHOl;H1O, AtPHOl;HI, AtPHOl and AtPHT1;1 in Pi-starved plants, while similar treatment induced $\mathrm{AtPHOI} ; \mathrm{HIO}$ and $\mathrm{AtPHOl} ; \mathrm{HI}$ expression and repressed AtPHOI expression in Pi-sufficient plants (Fig. 5b). Auxin addition to roots provokes modifications of the root architecture in high Pi medium similar to the one induced by Pi deficiency, such as alteration of primary root growth and promotion of root hair and lateral root formation (Torrey 1976). Furthermore, several studies have revealed complex interactions between auxin and the root architectural modifications due to phosphate starvation
(López-Bucio et al. 2002; Lopez-Bucio et al 2005; AlGhazi et al. 2003; Nacry et al. 2005; Jain et al. 2007). Lopez-Bucio et al. (2005) proposed the involvement of two different pathways in low phosphate stress-induced root architectural modifications where one is auxin dependent and the other auxin independent. Moreover, local changes in auxin concentrations and in auxin sensitivity in plant roots have been shown to be responsible for some specific alterations of root system architecture in adaptation to low Pi stress (Al-Ghazi et al. 2003; Lopez-Bucio et al. 2005; Nacry et al. 2005). At the gene expression level, addition of auxin has been shown to lead to variable expression pattern for Pi-starvation induced gene. Thus, while auxin addition did not influence expression of At4, AtIPS1, AtPHT1;4, AtRNAse 2 or OsIPS2 under Pi-deficient conditions, similar treatment either repressed the expression of AtPHT1;1 or induced the expression of OSIPS1 and AtMDG2 (Martin et al. 2000; Karthikeyan et al. 2002; Hou et al. 2005; Kobayashi et al. 2006; Shin et al. 2006). This heterogeneity in responses of gene expression may be potentially linked to the correspondence between local increase or decrease in auxin concentration or sensitivity in regions of the root or other tissues, with the distribution of the expression pattern of the various genes studied (Nacry et al. 2005). Importantly, the present study reveals that the effect of both auxin and cytokinin on the expression of Pistarvation induced gene is highly dependent on the Pi status of the plants, and that even for two genes, such as AtPHOl and AtPHOl;H1, which have similar functions in the loading of Pi to the root xylem vessels (Stefanovic et al. 2007), the effect of these hormones can be opposite in Pi-sufficient plants.

Compared to auxin and cytokinin, the role of ABA on plant adaptation to phosphate deficiency is less well defined. ABA treatment leads to a decrease in plant weight but an increase in the root-to-shoot ratio and root hair density comparable to the one occurring during phosphate deficient conditions, suggesting that ABA could also mediate some responses of the plant to Pi starvation (Watts et al. 1981). An increase in stomatal responsiveness to applied ABA has been measured in phosphorus-stressed plants, implying that sensitivity to ABA was altered by phosphorus stress (Radin 1984). Studies performed on Ricinus communis L. revealed that xylem transport of $\mathrm{ABA}$ in Pi-deficient plants was stimulated by a factor of 6 , whereas phloem transport was affected only very slightly (Jeschke et al. 1997; Jeschke and Hartung 2000). Moreover, it was reported that abi2-1 mutants accumulate less anthocyanin in response to Pi starvation (Trull et al. 1997) and that the expression of rab18 under Pi-starvation conditions is partially reduced in the abal mutant (Ciereszko and Kleczkowski 2002), suggesting that some degree of cross-talk between ABA and Pi-starvation signaling exists in plant. However, the 
comparison of growth and biochemical responses (acid phosphatase production in response to $\mathrm{Pi}$ deficiency) of A. thaliana ABA mutants $a b a-1$ and abi2-1 to those of wild-type plants indicated that ABA does not have a major role in coordinating the Pi deficiency response (Trull et al. 1997). More recently, ABA treatment has been shown to repress the induction of At4 and OsIPS1 and OsIPS2 in Pideficient plants (Hou et al. 2005; Shin et al. 2006). The present study reveals that in contrast to the effect of auxin and cytokinin, the effects of addition of exogenous ABA on gene expression were similar in Pi-sufficient and Pi-deficient plants, in that $\mathrm{ABA}$ repressed the expression of AtPHOl, AtPHOl;H1 and AtPHT1;1, but activated the expression of AtPHO1;H1O expression (Fig. 6a, c). This transcriptional regulation was further confirmed by modifying the endogenous level of ABA using the abal-3 mutant deficient in ABA synthesis (Fig. 6b). Importantly, the repression of AtPHOI, AtPHOI;H1, AtIPSI, and AtPHT1;1 expression by addition of exogenous ABA was dependent on ABI1, a type $2 \mathrm{C} \mathrm{Ser/Thr} \mathrm{protein} \mathrm{phosphatase} \mathrm{participat-}$ ing in numerous responses of plants to ABA during the germinative and post-germinative phases (Fig. 6c; Rock 2000). These results, thus, uncovered the participation of the ABA signal transduction cascade involving ABI1 in the regulation of several genes involved in Pi homeostasis, including AtPHOl, AtPHOI;H1, AtIPSI and AtPHTl;1.

In conclusion, this study shows that regulation of three homologues of the AtPHOl gene family by Pi deficiency involves the interaction between multiple-signaling pathways involving the plant Pi status as well as sucrose, auxin, cytokinin and ABA. The final outcome on gene expression from the integration of these parameters can sometimes be opposite, even for members of a gene family that have a similar function in Pi homeostasis, such as for AtPHOl and AtPHOl;H1, underlying the complexity of the plant's responses to Pi deficiency. Finally, ABA and the ABI1mediated signal transduction cascade has been implicated in the regulation of several genes responding to Pi starvation, and the impact of this hormone on Pi homeostasis and the adaptation of plants to Pi deficiency deserves further attention.

Acknowledgments This research was funded, in part, from a FNS grant (3100A0-105874) to YP, as well as from the Herbette Foundation and the Etat de Vaud. The authors are grateful to Javier Paz-Ares (Centro Nacional de Biotecnologia, Madrid) for providing seeds of $\mathrm{phrl}$ mutant and Hatem Rouached (University of Lausanne) for critical reading of the manuscript.

\section{References}

Al-Ghazi Y, Muller B, Pinloche S, Tranbarger TJ, Nacry P, Rossignol M, Tardieu F, Doumas P (2003) Temporal responses of Arabidopsis root architecture to phosphate starvation: evidence for the involvement of auxin signalling. Plant Cell Environ 26:10531066

Aung K, Lin S-I, Wu C-C, Huang Y-T, Su C-L, Chiou T-J (2006) pho2, a phosphate overaccumulator, is caused by a nonsense mutation in a miR399 target gene. Plant Physiol 141:1000-1011

Bari RP, Pant BD, Stitt M, Scheible W-R (2006) PHO2, micro RNA399 and PHR1 define a phosphate signalling pathway in plants. Plant Physiol 141:988-999

Chen Z-H, Nimmo GA, Jenkins GI, Nimmo HG (2007) BHLH32 modulates several biochemical and morphological processes that respond to Pi starvation in Arabidopsis. Biochem J 405:191-198

Chiou TJ, Aung K, Lin S-I, Wu C-C, Chiang S-F, Su C-L (2006) Regulation of phosphate homeostasis by microRNA in Arabidopsis. Plant Cell 18:412-421

Ciereszko I, Kleczkowski LA (2002) Effects of phosphate deficiency and sugars on expression of rab18 in Arabidopsis: hexokinasedependent and okadaic acid-sensitive transduction of the sugar signal. Biochim Biophys Acta 1579:43-49

Cieresko I, Johansson H, Hurry V, Kleczkowski LA (2001) Phosphate status affects the gene expression, protein content and enzymatic activity of UDP-glucose pyrophosphorylase in wild-type and pho mutants of Arabidopsis. Planta 212:598-605

Ciereszko I, Johansson H, Kleczkowski LA (2005) Interactive effects of phosphate deficiency, sucrose and light/dark conditions on gene expression of UDP-glucose pyrophosphorylase in Arabidopsis. J Plant Physiol 162:343-353

Crowe ML, Serizet C, Thareau V, Aubourg S, Rouze P, Hilson P, Beynon J, Weisbeek P, Van Hummelen P, Reymond P, Paz-Ares J, Nietfeld W, Trick M (2003) CATMA: a complete Arabidopsis GST database. Nucleic Acids Res 31:156-158

Delhaize E, Randall PJ (1995) Characterization of a phosphate-accumulator mutant of Arabidopsis thaliana. Plant Physiol 107:207-213

Devaiah BN, Karthikeyan AS, Raghothama KG (2007) WRKY75 transcription factor is a modulator of phosphate acquisition and root development in Arabidopsis. Plant Physiol 143:1789-1801

Ei-D AMSA, Salama A, Wareing PF (1979) Effects of mineral nutrition on endogenous cytokinins in plants of sunflower (Helianthus annus L.). J Exp Bot 30:971-981

Franco-Zorrilla JM, Martin AC, Solano R, Rubio V, Leyva A, PazAres J (2002) Mutations at CRE1 impair cytokinin-induced repression of phosphate starvation responses in Arabidopsis. Plant J 32:353-360

Franco-Zorrilla JM, Martin AC, Leyva A, Paz-Ares J (2005) Interaction between phosphate-starvation, sugar, and cytokinin signaling in Arabidopsis and the roles of cytokinin receptors CRE1/AHK4 and AHK3. Plant Physiol 138:847-857

Franco-Zorilla JM, Valli A, Todesco M, Mateos I, Puga MI, Rubio-Somoza I, Leyva A, Weigel D, Garcia JA, Paz-Ares J (2007) Target mimicry provides a new mechanism for regulation of microRNA activity. Nat Genet 39:1033-1037

Hamburger D, Rezzonico E, MacDonald-Comber Petétot J, Somerville C, Poirier Y (2002) Identification and characterization of the Arabidopsis $\mathrm{PHO1}$ gene involved in phosphate loading to the xylem. Plant Cell 14:889-902

Hammond JP, Bennett MJ, Bowen HC, Broadley MR, Eastwood DC, May ST, Rahn C, Swarup R, Woolaway KE, White PJ (2003) Changes in gene expression in Arabidopsis shoots during phosphate starvation and the potential for developing smart plants. Plant Physiol 132:578-586

Horgan JM, Wareing PF (1980) Cytokinins and the growth responses of seedlings of Betula pendula Roth. and Acer pseudoplatanus L. to nitrogen and phosphorus deficiency. J Exp Bot 31:525-532

Hou XL, Wu P, Jiao FC, Jia QJ, Chen HM, Yu J, Song XW, Yi KK (2005) Regulation of the expression of OsIPS1 and OsIPS2 in rice via systemic and local Pi signalling and hormones. Plant Cell Environ 28:353-364 
Jain M, Poling MD, Karthikeyan AS, Blakeslee JJ, Peer WA, Titapiwatanakun B, Murphy AS, Raghothama KG (2007) Differential effects of sucrose and auxin on localized phosphate deficiency-induced modulation of different traits of root system architecture in Arabidopsis. Plant Physiol 144:232-247

Jeschke WD, Hartung W (2000) Root-shoot interactions in mineral nutrition. Plant Soil 226:57-69

Jeschke WD, Peuke AD, Pate JS, Hartung W (1997) Transport, synthesis and catabolism of abscisic acid (ABA) in intact plants of castor bean (Ricinus communis L.) under phosphate deficiency and moderate salinity. J Exp Bot 48:1737-1747

Karthikeyan AS, Varadarajan DK, Mukatira UT, D’Urzo MP, Damsz B, Raghothama KG (2002) Regulated expression of Arabidopsis phosphate transporters. Plant Physiol 130:221-233

Karthikeyan AS, Varadarajan DK, Jain A, Held MA, Carpita NC, Raghothama KG (2006) Phosphate starvation responses are mediated by sugar signaling in Arabidopsis. Planta 225:907-918

Kobayashi K, Masuda T, Takamiya K-I, Ohta H (2006) Membrane lipid alteration during phosphate starvation is regulated by phosphate signaling and auxin/cytokinin cross-talk. Plant J 47:238-248

Koornneef M, Jorna ML, Brinkhorst-van der Swan DLC, Karssen CM (1982) The isolation of abscisic acid (ABA) deficient mutants by selection of induced revertants in non-germinating gibberellin sensitive lines of Arabidopsis thaliana (L.) Heynh. Theor Appl Genet 61:385-393

Koornneef M, Reiuling G, Karssen CM (1984) The isolation and characterization of abscisic acid insensitive mutants of Arabidopsis thaliana. Physiol Plant 61:377-383

Lai F, Thacker J, Li Y, Doerner P (2007) Cell division activity determines the magnitude of phosphate starvation responses in Arabidopsis. Plant J 50:545-556

Lejay L, Gansel X, Cerezo M, Tillard P, Mueller C, Krapp A, von Wiren N, Daniel-Vedele F, Gojon A (2003) Regulation of root ion transporters by photosynthesis: functional importance and relation with hexokinase. Plant Cell 15:2218-2232

López-Bucio L, Hernández-Abreu E, Sánchez-Calderón L, Nieto-Jacobo MR, Simpson J, Herrera-Estrella L (2002) Phosphate sensitivity alters architecture and causes changes in hormone sensitivity in the Arabidopsis root system. Plant Physiol 129:244-256

Lopez-Bucio J, Hernandez-Abreu E, Sanchez-Calderon L, Perez-Torres A, Rampey R, Bartel B, Herrera-Estrella L (2005) An auxin transport independent pathway is involved in phosphate stress-induced root architectural alterations in Arabidopsis. Identification of BIG as a mediator of auxin in pericycle cell activation. Plant Physiol 137:681-691

Martin AC, del Pozo JC, Iglesias J, Rubio V, Solano R, de la Peña A, Leyva A, Paz-Ares J (2000) Influence of cytokinins on the expression of phosphate starvation-responsive genes in Arabidopsis. Plant J 24:559-567

Misson J, Thibaud MC, Bechtold N, Raghothama K, Nussaume L (2004) Transcriptional regulation and functional properties of Arabidopsis Pht 1;4, a high affinity transporter contributing greatly to phosphate uptake in phosphate deprived plants. Plant Mol Biol 55:727-741

Misson J, Raghothama KG, Jain A, Jouhet J, Block MA, Bligny R, Ortet P, Creff A, Somerville S, Rolland N, Doumas P, Nacry P, Herrerra-Estrella L, Nussaume L, Thibaud MC (2005) A genomewide transcriptional analysis using Arabidopsis thaliana Affymetrix gene chips determined plant responses to phosphate deprivation. Proc Natl Acad Sci USA 102:11934-11939

Miura K, Rus A, Sharkhuu A, Yokoi S, Karthikeyan AS, Raghothama KG, Baek D, Koo YD, Jin JB, Bressan RA, Yun DJ, Hasegawa PM (2005) The Arabidopsis SUMO E3 ligase SIZ1 controls phosphate deficiency responses. Proc Natl Acad Sci USA 102:7760-7765

Morcuende R, Bari RP, Gibon Y, KZheng W, Datt Pant B, Bläsing O, Usadel B, Czechowski T, Udvardi MK, Stitt M, Scheible WR
(2007) Genome-wide reprogramming of metabolism and regulatory networks of Arabidopsis in response to phosphorus. Plant Cell Environ 30:85-112

Müller R, Nilsson L, Krintel C, Nielsen TH (2004) Gene expression during recovery from phosphate starvation in roots and shoots of Arabidopsis thaliana. Physiol Plant 122:233-243

Müller R, Nilsson L, Nielsen LK, Nielsen TH (2005) Interaction between phosphate starvation signalling and hexokinase-independent sugar sensing in Arabidopsis leaves. Physiol Plant 124:81-90

Müller R, Morant M, Jarmer H, Nilsson L, Hamborg Nielsen TH (2007) Genome-wide analysis of the Arabidopsis leaf transcriptome reveals interaction of phosphate and sugar metabolism. Plant Physiol 143:156-171

Nacry P, Canivenc G, Muller B, Azmi A, Van Onckelen H, Rossignol M, Doumas P (2005) A role for auxin redistribution in the responses of the root system architecture to phosphate starvation in Arabidopsis. Plant Physiol 138:2061-2074

Nielsen TH, Krapp A, Roeper-Schwarz U, Stitt M (1998) The sugarmediated regulation of genes encoding the small subunit of Rubisco and the regulatory subunit of ADP glucose pyrophosphorylase is modified by phosphate and nitrogen. Plant Cell Environ 21:443-454

Poirier Y, Bucher M (2002) Phosphate transport and homeostasis in Arabidopsis. In: Somerville CR, Meyerowitz EM (eds) The Arabidopsis book. American Society of Plant Biologists, Rockville

Poirier Y, Thoma S, Somerville C, Schiefelbein J (1991) A mutant of Arabidopsis deficient in xylem loading of phosphate. Plant Physiol 97:1087-1093

Radin JW (1984) Stomatal responses to water stress and abscisic acid in phosphorus deficient cotton plants. Plant Physiol 76:392-394

Raghothama KG (2000) Phosphate transport and signaling. Curr Opin Plant Biol 3:182-187

Rock CD (2000) Pathways to abscisic acid-regulated gene expression. New Phytol 148:357-396

Rubio V, Linhares F, Solano R, Martin AC, Iglesias J, Leyva A, PazAres J (2001) A conserved MYB transcription factor involved in phosphate starvation signaling both in vascular plants and in unicellular algae. Genes Dev 15:2122-2133

Shin H, Shin HS, Dewbre GR, Harrison MJ (2004) Phosphate transport in Arabidopsis: Pht1;1 and Pht1;4 play a major role in phosphate acquisition from both low-and high-phosphate environments. Plant J 39:629-642

Shin H, Shin H-S, Chen R, Harrison MJ (2006) Loss of At4 function impacts phosphate distribution between the roots and the shoots during phosphate starvation. Plant J 45:712-726

Stefanovic A, Ribot C, Rouached H, Wang Y, Chong J, Belbahri L, Delessert S, Poirier Y (2007) Members of the PHOl gene family show limited functional redundancy in phosphate transfer to the shoot, and are regulated by phosphate deficiency via distinct pathways. Plant J 50:982-994

Svistoonoff S, Creff A, Reymond M, Sigoillot-Claude C, Ricaud L, Blanchet A, Nussaume L, Desnos T (2007) Root tip contact with low-phosphate media reprograms plant root architecture. Nat Genet 39:792-796

Ticconi CA, Delatorre CA, Abel S (2001) Attenuation of phosphate starvation responses by phosphite in Arabidopsis. Plant Physiol 127:963-972

Torrey JG (1976) Root hormones and plant growth. Annu Rev Plant Physiol Plant Mol Biol 27:435-459

Trull MC, Guiltinan MJ, Lynch JP, Deikman J (1997) The responses of wild-type and ABA mutant Arabidopsis thaliana plants to phosphorus starvation. Plant Cell Environ 20:85-92

Uhde-Stone C, Zinn KE, Ramirez-Yanez M, Li A, Vance CP, Allan DL (2003) Nylon filter arrays reveal differential gene expression in proteoid roots of white lupin in response to phosphorus deficiency. Plant Physiol 131:1064-1079 
Varadarajan DK, Karthikeyan AS, Matilda PD, Raghothama KG (2002) Phosphite, an analogue of phosphate, suppresses the coordinated expression of genes under phosphate starvation. Plant Physiol 129:1232-1240

Wang YH, Garvin DF, Kochian LV (2002) Rapid induction of regulatory and transporter genes in response to phosphorus, potassium, and iron deficiencies in tomato roots. Evidence for cross talk and root/rhizosphere-mediated signals. Plant Physiol 130:1361-1370

Wang Y, Ribot C, Rezzonico E, Poirier Y (2004) Structure and expression profile of the Arabidopsis $\mathrm{PHOl}$ gene family indicates a broad role in inorganic phosphate homeostasis. Plant Physiol 135:400-411
Wang X, Yi K, Tao Y, Wang F, Wu Z, Jiang D, Chen X, Zhu L, Wu P (2006) Cytokinin represses phosphate-starvation response through increasing of intracellular phosphate level. Plant Cell Environ 29:1924-1935

Watts S, Rodriguez J, Evans S, Davies W (1981) Roots and shoot growth of plants treated with abscisic acid. Ann Bot 47:595-602

Wu P, Ma L, Hou X, Wang M, Wu Y, Liu F, Deng XW (2003) Phosphate starvation triggers distinct alterations of genome expression in Arabidopsis roots and leaves. Plant Physiol 132:1260-1271

Yi K, Wu Z, Zhou J, Du L, Guo L, Wu Y, Wu P (2005) OsPTF1, a novel transcription factor involved in tolerance to phosphate starvation in rice. Plant Physiol 138:2087-2096 